Wenner, D.M. (2015). “Barriers to Effective Deliberation in Clinical Research Oversight,” HEC Forum, doi: 10.1007/s10730-015-9298-0 (epub ahead of print).

This is a pre-print of a published manuscript. When citing, please refer to the final, published version at http://link.springer.com/article/10.1007\%2Fs10730-015-9298-0

Address correspondence to:

Danielle M. Wenner

Department of Philosophy, Carnegie Mellon University

155C Baker Hall, Pittsburgh, PA 15213

Telephone 412-268-8046

FAX 412-268-1440

dwenner@andrew.cmu.edu 


\section{Barriers to Effective Deliberation in Clinical Research Oversight}

Ethical oversight of clinical research is one of the primary means of ensuring that human subjects are protected from the natural bias of researchers and research institutions in favor of experimentation. Such oversight can also help to buttress public trust in a system of evidence production that depends largely on the ongoing support of stakeholders with a wide range of personal motives for contributing to its continued existence (London 2012). When operating well, ethical oversight therefore plays an important role in the health research ecosystem.

Properly functioning institutional review boards (IRBs) should approve ethical and valid studies and disapprove unethical or invalid studies. However it's not always clear what the mandate of IRBs to approve all and only ethical and valid studies entails. At a minimum, effective oversight should ensure that risks are minimized and reasonable in relation to anticipated benefits, protect vulnerable research subjects from potential coercion or undue influence, ensure full and informed consent, and promote the equitable distribution of the risks and benefits of research (U.S. Department of Health and Human Services 2009, §111). Because these assessments often involve value judgments for which there are no agreed-upon objective standards, we depend on specific procedures thought to have the greatest likelihood of producing the right or best outcomes.

In the case of IRBs, we rely on a deliberative procedure among a diverse group of participants from varying backgrounds and expertise. The hope is that by including members with various perspectives, all relevant considerations will be brought to bear on discussion. In an ideal deliberative procedure, rational consensus is sought via individual parties’ full and equal abilities to raise issues and influence discussion, and outcomes are based on reasons offered in support of particular decisions rather than inequalities of power or bargaining position (Cohen 
[1989] 1997; Habermas 1996). In order to approximate this ideal, IRBs must not only be staffed by sufficiently qualified and diligent members; protocol deliberations must also be conducted in a manner that ensures all relevant input is heard and given adequate consideration.

When IRBs fail to function properly, they not only waste scarce human and institutional resources, they also threaten to impede the pace of biomedical progress. In preventing sound and ethical research from being conducted or approving research that is ethically or methodologically unsound, IRBs can contribute to the diversion of participants, institutional resources, and limited funding away from important studies to trials that are unlikely to generate socially valuable knowledge. This threat has led some researchers to accuse IRBs of stifling progress and creativity (Hamburger 2007), discouraging good research (Gunsalus et al. 2006), impeding biomedical progress (Epstein 2007), misdirecting scarce resources (Gunsalus et al. 2006; Whitney et al. 2008), and even possibly encouraging research misconduct (Keith-Spiegel and Koocher 2005).

Such concerns have motivated a recent increase in the volume of studies seeking to assess the procedures and outcomes of oversight boards. Recent empirical research has examined several dimensions of IRBs governing clinical research. Studies have sought to characterize the composition of IRBs (Schuppli and Fraser 2007), differences in IRB process (Larson et al. 2006), the nature and cause of outcome differentials (Abbott and Grady 2011; Klitzman 2011), how IRBs interact with their broader institutional settings (Klitzman 2013c), the extent to which IRBs successfully apply the Common Rule federal regulations for ethical research (Lidz et al. 2012a), as well as how IRB members perceive and address a variety of different issues that arise during prospective review (Klitzman 2012b, 2013a, 2013b). Such research helps to highlight where oversight processes may break down or have room for improvement. 
Among the myriad empirical studies assessing IRB oversight, however, there has been minimal attention paid to the substantial body of social scientific research regarding the ways that individuals and deliberating groups make decisions, and how those data might inform IRB practice. This review essay seeks to fill that gap, locating recent empirical data on IRB composition and process within the context of data regarding what I call "deliberative pathologies." Specifically, I use this term to refer to instances when deliberation fails to live up to one or more aspect of the deliberative ideal because of systematic biases in the ways participants interact. Such pathologies can be due to differences in linguistic cultures, individual differences in personality and ability, and even individually rational behaviors that nevertheless function to deprive deliberation of some of its value in soliciting various perspectives. Insofar as such pathologies impact IRBs' abilities to approximate the ideal deliberative procedure, there is reason to explore evidence-based approaches to reducing the impacts of deliberative pathologies on oversight deliberation.

This paper is limited in scope to a convenience sample of three specific forms of deliberative pathology and their potential manifestation in clinical research oversight. In particular, the potential impacts of epistemic and linguistic biases, personality effects, and polarization and sequence effects on deliberative decision-making are discussed. Both empirical evidence of the prevalence of these pathologies as well as theoretical accounts of their potential causes are leveraged to illustrate how deliberative pathologies can undermine the goals of deliberative decision-making. Recent empirical evidence regarding the constitution, processes, and interactions of IRBs is used to demonstrate the vulnerability of current practices to these pathologies. The paper goes on to make evidence-based recommendations to reduce the 
vulnerability of IRB deliberations to the three kinds of pathologies discussed. Finally, directions are indicated in which future research might be fruitful.

\section{Epistemic and Linguistic Biases}

United States federal regulations mandate IRB membership of a minimum of five members, including at least one scientist member (such as a physician, investigator, or statistician), at least one non-scientist member (such as an ethicist), and at least one member not directly affiliated with the institution (i.e. not employed by the institution or an immediate family member of someone who is). The same individual can play more than one role; so for example, a nonscientist member might also be unaffiliated with the institution. The regulations also stipulate that the board be sufficiently diverse with regards to race, gender, and sensitivity to community attitudes (U.S. Department of Health and Human Services 2009). Similar regulations govern research ethics committees in other countries: the UK’s Health Research Authority requires a minimum of seven members, with at least one lay member and one “expert” member (National Health Service Health Research Authority 2012), while CIOMS guidelines recommend that “membership should include physicians, scientists and other professionals such as nurses, lawyers, ethicists and clergy, as well as lay persons qualified to represent the cultural and moral values of the community” (Council for International Organizations of Medical Sciences (CIOMS) 2002).

Requirements for multidisciplinary and representative membership are predicated on two ideas. First, those with different areas of expertise and viewpoints can introduce unique perspectives into the oversight process (E. E. Anderson 2006). For example, the inclusion of non-affiliated members, often referred to as “community members”, is hoped to provide insight into the concerns of local populations from which study participants will be drawn. Similarly, 
requiring inclusion of members without institutional affiliations should help to balance the financial and other professional interests that institutional members may have in seeing research approved. More broadly, non-scientists may help to introduce considerations that correct for the bias in favor of experimentality that is otherwise likely to dominate a board comprised entirely of physician-scientists. Second, a diverse and representative membership can serve to buttress public support for the oversight process, providing greater confidence that a board is representative of community needs and therefore better equipped to ensure the protection of research participants from harm or exploitation (Allison et al. 2008). Given the important roles played by this diverse membership, it would be reason for concern if some voices were systematically neglected or overlooked in oversight deliberations.

Yet as empirical research on the process and function of IRBs proliferates, data is mounting that non-affiliated and non-scientist members are in many cases not participating as effectively as might be necessary to fill the important roles envisaged for them. For example, non-scientist and non-affiliated members have reported in multiple studies feeling either intimidated by other members of their boards or that other members perceived them to have insufficient expertise (Porter 1986; Sengupta and Lo 2003; Schuppli and Fraser 2007; Klitzman 2012a).

The accuracy of these feelings is borne out by data collected on the participation of nonaffiliated and non-scientist members on IRBs. Although there is a great deal of variability with regard to what roles and responsibilities such members are assigned in oversight (Porter 1987; Klitzman 2012a), non-affiliated and non-scientist members were found in one study to constitute a very small minority of primary reviewers, and a minority of secondary and tertiary reviewers as well (Lidz et al. 2012b). This suggests that although boards may look to non-affiliated and non- 
scientist members for comment on specific topics, such as informed consent forms, those members are overall accorded significantly less input into deliberations. Insofar as they are not assigned as primary or secondary reviewers, it is also likely that what input non-affiliated and non-scientist members do contribute may be accorded less epistemic weight than contributions from others. The one outlier in this regard was a study by Allison et. al. (2008) conducted among non-scientist members on National Institutes of Health (NIH) IRBs. This team found that non-scientist members felt empowered to speak and that their contributions were accorded appropriate weight, but this may be attributable to features unique to the NIH, including access to a prominent in-house bioethics program from which to draw non-scientists who nevertheless hold significant institutional credentials.

Some of this deficit in epistemic credence may be due to actual gaps in understanding or expertise. But insofar as this lack of credence is due to causes attributable to the status of nonaffiliated and non-scientist members rather than the quality of their input, IRBs are failing to make proper use of the resources available to them, and may be doing so to the detriment of their ability to serve their putative goals of protecting research subjects, ensuring ethical research, and taking into account the values and perspectives of the communities from which their subjects are drawn.

In order to be effective in group deliberations, individual members must be capable of communicating their ideas clearly and persuasively to the rest of the group. Discourse ethicists have theorized that a crucial part of this effectiveness is the ability to present opinions and arguments via means of communication accorded epistemic weight by other members of the group. This includes particular vocabularies, idioms, narratives, and argumentative strategies which the dominant group accepts as authoritative (Fraser 1995). The physicians, researchers, 
and scientists who comprise the majority on most IRBs are fluent in a particular specialist language, while non-affiliated and non-scientist members may not be. In some cases, nonaffiliated or non-scientist members may in fact have significant experience with medical research and thus a level of fluency with this dominant linguistic culture, such as 2 "community members” identified in a study by Lidz et. al. (2012b) who were a pediatric nurse with several years' experience working on an IRB and a retired hospital administrator. It is unclear how prevalent this may be, but insofar as diverse representation on IRBs is intended to correct for biases in favor of research and to ensure that community views which diverge from institutional perspectives are represented, the use of such individuals as non-affiliated members may ultimately undermine that goal. Meanwhile, those non-scientist and non-affiliated members without this level of medical background may find themselves lacking a certain kind of privilege in oversight deliberations. In one study of IRBs, non-affiliated board members who reported feeling intimidated by other members during their service frequently associated this feeling with their own unfamiliarity with technical terms, jargon, and the clinical milieu with which their colleagues on the board were much better versed (Porter 1986).

That said, the dominant linguistic culture need not be construed as limited to certain vocabularies or rhetorics. It can include forms of gesticulation or nuances in speech patterns that often vary across social and economic classes (Fraser 1995). This means that even when issues under discussion veer away from the scientific and into worries such as obtaining adequate consent or social value, non-affiliated members may find their input unconsciously discounted on the basis of their linguistic habits rather than on the basis of the content of their contributions. Or, if such members attempt to communicate using the linguistic norms of the scientist members of the board, their lesser fluency in that linguistic form may reduce their influence or credibility. 
Research on this phenomenon suggests that minority participants will more successfully communicate in the dominant linguistic paradigm when discussions feel unthreatening, which in turn causes other members of the group to perceive them as "cooperative, friendly, and effective.” But when threat levels are perceived to be high, those in the linguistic minority will abandon the majority's linguistic norms and overall levels of productive cooperation will decrease (Delli Carpini et al. 2004).

What these kinds of linguistic biases demonstrate is the potential for an inequality of deliberative influence between scientist and institution-based members on the one hand, and nonscientist and non-affiliated members on the other. To the extent that this this inequality traces to membership in the dominant linguistic class rather than to the quality of the reasons on offer, non-affiliated and non-scientist IRB members may be unable to perform their roles even when otherwise well-qualified to do so.

The inability to deliberate effectively in the dominant language not only contributes to the discounting of input by non-scientist and non-affiliated members, but can also affect their standing in the group hierarchy. Multiple studies demonstrate that deliberators already tend to give the least weight to the input of perceived "low-status" members, which within an institutional setting may simply be those members not recognized as professional peers (Christensen and Abbott 2000). Insofar as lack of fluency with dominant linguistic norms can be used as a proxy for the status of deliberators, linguistic norms take on added importance. The tendencies of oversight boards to be comprised of a majority of institution-based physicianscientist members (Abbott and Grady 2011) and to be predominantly composed of white males (De Vries and Forsberg 2002) both ensure that such boards are particularly vulnerable to this form of bias. 
Linguistic bias can also manifest in small differences in the ways that group members communicate with and about one another and each other's input. When a group is characterized by a majority, or "in-group”, and a minority, or “out-group”, members of the in-group tend to refer to the contributions of different parties in subtly different ways. For example, when an ingroup member contributes something helpful to discussion, other members of the in-group will use an abstract term to refer to them, such as "helpful", whereas if that same contribution is made by an out-group member, in-group members are more likely to use concrete terms such as "helping". The use of abstract versus concrete descriptors reverses in the case of unhelpful or distracting input: in-group members' contributions will be described in concrete terms such as “harming”, while out-group members' contributions will be assigned abstract merit, such as "harmful" (Maass et al. 1996). This small linguistic difference - known as "linguistic intergroup bias" - communicates that the positive contributions of in-group members (and the negative contributions of out-group members) are due to inherent qualities, since abstract terms such as "helpful" and "harmful" carry connotations about individuals as well as actions. Meanwhile, the use of concrete terms such as "helping" and "harming" to characterize in-group members' negative contributions (and out-group members' positive) suggests that any association with the individuals are merely accidental, since such descriptors more accurately apply to individual actions, rather than to the agent herself. This introduction of stereotypes into linguistic norms has been consistently demonstrated in empirical studies (Mendelberg 2002). Such subtle differences reinforce the kinds of epistemic inequalities generated by unfamiliarity with dominant linguistic norms and function to undermine the credibility of out-group members while reinforcing the hierarchical status of members of the in-group. 
While the role of non-affiliated and non-scientist members on IRBs has long been a matter of debate, absent some threshold level of epistemic authority in deliberation their presence stands to contribute little to research oversight. Without credibility for their input, non-affiliated and non-scientist members cannot function as a check on institutional interests, nor represent the communities from which participants are drawn. Effective oversight procedures should therefore include processes for promoting and protecting the influence and credibility of non-affiliated and non-scientist members.

\section{Personality Effects}

A related pathology involves the impacts that individual differences in personality can have on contributions to, and outcomes of, deliberation. Remember that when functioning properly, deliberation should offer participants equal opportunities to provide input and to influence decision-making outcomes. Moreover, those outcomes should be based on the quality of the reasons and arguments offered in support of particular decisions, rather than inequalities of bargaining power or other features that are irrelevant to the decision itself.

Actual deliberative processes often fall far short of this deliberative ideal. In addition to the loss of influence that some participants suffer due to linguistic biases, certain personality traits will more often than not dominate discussions, have greater influence on other participants, and gain significant control over group interactions (C. Anderson and Kilduff 2009).

Extroversion in particular is the most consistent predictor of the assumption of leadership roles in group discussions (Judge et al. 2002). This might not be problematic, if less outgoing personalities were nevertheless able to influence deliberative outcomes on the basis of strong reasons and persuasive arguments. But in addition to their tendency to assume leadership roles, those who self-identify as "extroverted" are also significantly less likely to revise their pre- 
deliberative opinions on the basis of discussion and debate than those who self-identify as “introverted”, even when confronted with the same information (Gastil et al. 2008).

Insofar as board members with strong personalities are more likely to drive the direction of debate while introverts are more likely to refrain from offering input, oversight discussion has an even greater potential to omit the potentially valuable input of specific board members. This concern is amplified with regard to non-affiliated and non-scientist members, who may already wield less influence due to the linguistic dynamics discussed above. In one survey, 87 out of 198 unaffiliated IRB members (44\%) felt that in order to perform well, unaffiliated members needed to be assertive, self-confident, and courageous (Porter 1986). In another, some non-scientist members suggested that unaffiliated board members would be more likely to have their voices heard in meetings if only confident, outgoing individuals were selected to serve (Schuppli and Fraser 2007). This suggests both that dominant personality types do in fact play a significant role in IRB deliberations, as well as that the input of some members may be going unheard. To the extent that the unheard voices on IRBs are more likely to be those of non-affiliated or nonscientist members, this finding reinforces concerns raised in the previous section.

\section{Polarization and Sequence Effects}

Finally, in addition to the kinds of pathologies that result from role- and personality-specific variations in influence, we ought also to be concerned about the tendency of group deliberation to be pushed in the direction of the majority's pre-deliberative baseline. If the best method for determining the ethical bona fides of a clinical trial was a mere aggregation of the views of those who reviewed it, IRB oversight could be far more efficiently conducted by an independent review by all board members followed by a vote. The purpose of discussion on oversight boards is to make use of the varied knowledge and perspectives of individual members to ensure that 
outcomes track ethically and methodologically relevant criteria. But group deliberation is often pre-disposed towards outcomes that reflect the initial majority position in problematic ways.

In part, this is largely rational. When an individual deliberator finds her initial opinions to be reinforced by others who express the same or similar views, it is natural that her confidence in that view will grow, often resulting in the adoption of greater certainty or a more extreme position. Similarly, when multiple deliberators have expressed opinions in contradiction to that held by an individual, she is far less likely to voice dissent. This can be due not only to the social barriers presented by perceived conflict, but also because of a rational tendency to discount the value or accuracy of one's own knowledge when it is contradicted by several others in positions of epistemic authority. The result of these perfectly rational individual tendencies is a counter-productive group tendency to discount the validity or importance of alternative opinions when the majority already agrees, leaving outliers with greatly reduced influence over discussion and decision-making outcomes (C. Sunstein 2003).

Additionally, members who are "cognitively central” - i.e. those who share more reasons or arguments in common with other members of a group - tend to have the greatest influence over a group’s decisions, "regardless of their competence or the quality of their arguments" (Mendelberg 2002, 164). Such effects are most pronounced in highly cohesive groups that include a discrete minority, and when discussions center on difficult or nuanced problems as opposed to questions or issues with clear and discrete answers. In such settings - precisely the kinds of groups and decisions that characterize ethical oversight - deliberating groups have been shown to far under-perform a mere statistical aggregation of members' original, pre-deliberation preferences when outcomes can be measured against a procedure-independent standard of assessment (C. Sunstein 2006). 
Some of these effects can be due in part to the order in which deliberators speak, which can significantly impact both the willingness of later speakers to share unique or conflicting information, as well as the ultimate outcomes of decision-making (Austen-Smith 1995). When one position has been expressed by multiple members of a group, there is a significantly lower probability of others voicing dissent. Instead of members with new or conflicting information speaking up, deliberators are far more likely to speak in support of or reiterating points that have already been made. This both reinforces to those of the majority position that there are no reasonable objections to their perspective, while simultaneously motivating an even greater unwillingness to credit opposing information that is presented, and a failure to consider some novel information that is never introduced into deliberation (C. R. Sunstein 2000).

Many (perhaps most) IRBs delegate new and continuing protocol reviews to primary (and sometimes secondary or tertiary) reviewers in order to reduce the workload on the entire board. Primary reviewers are then often tasked with introducing a protocol and highlighting those issue upon which the committee might begin by focusing. In many cases, primary reviewers may be the only members to have closely examined a protocol, and are frequently in significant control of the framing of the discussion that follows as a result of having the opportunity to introduce a narrative frame first (Fitzgerald et al. 2006). Insofar as committees manifest pathologies related to sequence effects or information cascades, the "primary reviewer" model may exacerbate the effects by privileging one particular perspective in discussion. This effect will be amplified to the extent that certain members - such as non-affiliated and non-scientist members, as mentioned above - are systematically excluded from assignment as primary or secondary reviewers (c.f. page 5 and Lidz et al. 2012b). 
Another study, observing interactions on 20 IRBs across 10 high-volume medical research centers found that although two or more non-reviewers spoke during greater than $65 \%$ of protocol reviews observed, "their comments were typically brief and emphasized points [already] made by previous speakers” (Candilis et al. 2012, 17 [emphasis added]). This same study found that protocol discussions were largely dominated by IRB chairs and primary reviewers. Close to $15 \%$ of reviews were characterized by no input from other board members, and across boards anywhere from $6.7 \%$ to $44.4 \%$ of members said nothing for the entire duration of a meeting. Most telling, when IRB chairs expressed their opinions at the beginning of a discussion, a greater proportion of board members remained silent during the ensuing discussion.

The upshot is that a process designed to bring multiple, divergent perspectives to bear on difficult and nuanced problems can very easily become one that instead overlooks or marginalizes precisely those outlier views to which it is intended to give voice. This suggests that efforts should be made to minimize the vulnerability of oversight deliberation to the kinds of pathologies discussed here. The next section considers potential approaches to this task.

\section{Recommendations for Future Research}

To the extent that IRB oversight is intended to produce the "best" outcomes in terms of tracking risks and benefits, ensuring full and informed consent, protecting vulnerable populations, and ensuring a fair distribution of the burdens and benefits of research, the tendencies canvassed here should be of concern. The impacts of arbitrary features of deliberation and deliberators should be minimized, while competing ideas and inputs should be protected so as to ensure that all members' views have an opportunity to be brought to bear on deliberation. If particular features of IRB deliberations can be targeted for intervention to improve the quality of research oversight, such avenues warrant exploration. 
Two natural targets for further investigation are board size and board composition. Both the size and diversity of deliberating groups have been shown to impact the extent of pathological effects such as polarization and sequence effects, in that participation in larger groups has a tendency to amplify these effects while increases in diversity have a tendency to dampen them. For example, individual deliberators are more inclined to participate responsibly in group deliberations for which they feel accountable (Ryfe 2005), and individuals tend to feel less responsible for the outcomes of larger group deliberations (Latané and Wolf 1981). Similarly, dominant personalities tend to dominate more as group size increases and introverted members feel greater pressure to conform. The Candilis (2012) study cited above found an inverse correlation between the number of members present at board meetings and the proportion who actually participated in discussion. This study also found that more than half of the highvolume IRBs in their sample had 15 or more members, with a high of 44 . But the median participation was only 16 members. Correlations between group size and severity of deliberative pathologies suggest that one key strategy to improve oversight may be to reduce IRB membership.

In high volume centers, reduction of board size may require the addition of more boards, with an attendant increase in the administrative workload and costs associated with managing multiple IRBs. Moreover, as medical research becomes more specialized and technologically sophisticated, the need to enlist more experts who can shed light on protocols may increase. However, the continued maintenance of excessively large boards involves significant hidden costs when large proportions of members devote time and energy to protocol review and then contribute minimally to deliberations. The reduction of these costs may well offset a corresponding growth in administrative expenses. 
Alternatively, research institutions might consider maintaining larger boards with more representative expertise, but utilizing rotating attendance to keep discussions smaller, encourage more participation, and demand less time from members who are unlikely to have additional input for a given set of protocols. Ad hoc substitutes and consultants can be used to supplement regular membership and inform reviews of highly specific or technical protocols without inflating board size. Fruitful future research might compare participation rates and quality across boards of different sizes to determine whether limiting membership might improve the quality of deliberation or the tendency of outliers to express divergent opinions. Alternatively, the effects of board size on participation might be assessed through observation of member contributions on a single board across multiple meetings as attendance varies.

Similarly, polarization and domination are both reduced when heterogeneity within groups can be increased (C. R. Sunstein 2000), suggesting that greater study of board composition is warranted. This might go hand in hand with reduction in board size, thereby increasing the proportion of non-affiliated and non-scientist members. Alternatively, board size can be increased via the purposeful addition of non-affiliated and non-scientist members. Rothstein and Phuong (2007) argue that increasing non-affiliated membership will have no impact on deliberation, as non-affiliated and non-scientist members are no more likely to focus on ethical issues than other types of board members. However the goal of recruiting more nonaffiliated and non-scientist members need not be to "increase IRB concern with ethical issues" (Rothstein and Phuong 2007, 75). Rather, it can be to empower non-affiliated and non-scientist members to express their views with more confidence and to have those views accorded greater epistemic weight by others in the group. 
Research on the effectiveness of corporate boards has found mixed success with respect to increasing lay membership or bringing in outside leadership absent additional changes, but this has largely been attributed to the fact that outside members often serve at the request of institutional members and may feel beholden to them (Saver 2005). Indeed, in a study to assess perceptions of non-affiliated members on IRBs in India, Kuyare et. al. (2015) found that all 30 of the participants they surveyed were appointed via a contact person in the IRB, while no applications were invited by means of a public announcement. Klitzman (2012a) similarly found that a good number of non-affiliated and non-scientist members were health care professionals or former employees of the institutions for which they now consulted as IRB members. Thus one way to address board composition more effectively may be for research centers to explore means of recruiting unaffiliated members with fewer institutional, professional, or social ties to the institution or existing members. Boards can also increase the racial, ethnic, and gender diversity of their ranks as a means of encouraging more varied participation and guarding against the effects of in-group/out-group biases and polarization.

It is also worth exploring the impacts that formalized deliberative norms might have on oversight discussions. The persuasive strength of individual parties has been found to be bolstered by norms governing the process and structure of discussion in joint deliberations of international treaties (Ulbert and Risse 2005). Such norms might take the shape of directives regarding order of speech and the length or type of comments permitted at given points in deliberation, with particular attention to the duration and content of opening comments offered by primary reviewers or committee chairs. Specific tasks or roles might be assigned to different reviewers, with special attention to the role of non-affiliated and non-scientist members in discussion (Lidz et al. 2012b). Assigning non-affiliated members leadership roles could 
significantly buttress their deliberative influence, both by raising their perceived status and by increasing the likelihood that they will speak earlier during discussions (Ryfe 2005). A less conservative approach might give non-scientist and non-affiliated members an opportunity to deliberate together about protocols outside of the presence of the rest of the board (Saver 2005).

Efforts to diversify and introduce formal deliberative norms can be buttressed by educational efforts targeting better understanding of DHHS regulation, institutional policy, and relevant scientific and ethical issues. In a series of interviews with non-affiliated and nonscientist IRB members, Sengupta and Lo (2003) found that $47 \%$ cited lack of education and training as a problem, and nearly $80 \%$ felt that more in-depth training would be beneficial. Similarly, in a series of interviews with IRB chairs, administrators, and members, Klitzman (2013c) found that many IRB members saw differences in the level of knowledge and expertise of members, and felt that many would benefit from additional training. Such education can function not only to increase the confidence of unaffiliated and non-scientist members, and therefore their likelihood of contributing to discussions, but also to improve competence in the dominant linguistic culture. Greater proficiency with in-group linguistic culture will elevate the status of non-affiliated members and consequently strengthen their abilities to influence the outcomes of oversight deliberations and to promote the protection of research subjects. A preliminary step in this direction might be an exploration of the methods available for increasing IRB members' education, along with an assessment of associated costs.

Finally, further evidence regarding the effects of service length on deliberations within oversight boards is needed. Membership term lengths can directly impact the assumption of leadership roles, familiarity with the dominant linguistic culture, and in-group membership status. Future research on IRB composition should pay special attention to this feature with an 
eye towards answering two questions. First, it would be helpful to know whether limited terms of service for institution-based scientist members or board chairs might increase the willingness of board members to express dissenting opinions. And second, might increased terms of service for unaffiliated members increase their linguistic competence and consequently their perceived standing within the group, confidence levels, and ability to productively contribute to discussions? In researching the latter question, however, it will be important to address the tension between longer terms of service and the ability to appropriately reflect a non-institutional perspective (Schuppli and Fraser 2007).

\section{Conclusion}

IRB oversight is costly in terms of financial and human resources, and can introduce significant delays to biomedical progress. As empirical research on IRB process and function becomes more commonplace, institutions have the opportunity to examine and improve upon the quality of their oversight procedures, thus maximizing their returns on the associated costs. This review has sought to introduce the problem of deliberative pathologies to those interested in improving the quality and efficiency of IRB oversight. The discussion is limited in scope not only to a subset of potential pathologies, but also with regard to the evidence that can currently be brought to bear in recommendations for reform.

It is my hope that this survey will be of value in at least three ways: first, by providing motivation for those working in empirical research to more directly explore the impacts of deliberative pathologies on clinical research oversight; second, by offering a useful roadmap of potential future research directions of value in reducing the vulnerability of oversight procedures to these pathologies; and third, by highlighting the potential value of the vast social scientific 
literature on deliberation to those tasked with maximizing the effectiveness of oversight within clinical research institutions.

Future research should explore the potential to improve deliberative procedures via changes in board size, composition, and procedural norms, and should continue to make use of data generated within the fields of behavioral economics and deliberative democracy. To the extent that the manifestation of deliberative pathologies within clinical research oversight can be minimized and their effects controlled, IRB oversight can become more effective, more efficient, and less costly to research institutions and researchers themselves. 


\section{Works Cited}

Abbott, L., \& Grady, C. (2011). A Systematic Review of the Empirical Literature Evaluating IRBs: What We Know and What We Still Need to Learn. Journal of Empirical Research on Human Research Ethics, 6(1), 3-19.

Allison, R. D., Abbott, L. J., \& Wichman, A. (2008). Nonscientist IRB Members at the NIH. IRB: Ethics and Human Research, 30(5), 8-13.

Anderson, C., \& Kilduff, G. J. (2009). Why Do Dominant Personalities Attain Influence in Faceto-Face Groups? The Competence-Signaling Effects of Trait Dominance. Journal of Personality and Social Psychology, 96(2), 491-503.

Anderson, E. E. (2006). A Qualitative Study of Non-Affiliated, Non-Scientist Institutional Review Board Members. Accountability in Research, 13(2), 135-155.

Austen-Smith, D. (1995). Modeling Deliberative Democracy. Paper presented at the Workshop on Deliberative Democracy, University of Chicago.

Candilis, P. J., Lidz, C. W., Appelbaum, P. S., Arnold, R. M., Gardner, W., Myers, S., et al. (2012). The Silent Majority: Who Speaks at IRB Meetings? IRB: Ethics and Human Research, 34(4), 15-19.

Christensen, C., \& Abbott, A. S. (2000). Team Medical Decision Making. In G. B. Chapman, \& F. A. Sonnenberg (Eds.), Decision Making in Health Care: Theory, Psychology, and Applications (pp. 267-285). Cambridge: Cambridge University Press.

Cohen, J. ([1989] 1997). Deliberation and Democratic Legitimacy. In J. Bohman, \& W. Rehg (Eds.), Deliberative Democracy: Essays on Reason and Politics (pp. 67-91). Cambridge: Harvard University Press.

Council for International Organizations of Medical Sciences (CIOMS) (2002). International Ethical Guidelines for Biomedical Research Involving Human Subjects.

De Vries, R., \& Forsberg, C. P. (2002). Who Decides? A Look at Ethics Committee Membership. HEC Forum, 14(3), 252-258.

Delli Carpini, M. X., Cook, F. L., \& Jacobs, L. R. (2004). Public Deliberation, Discursive Participation, and Citizen Engagement: A Review of the Empirical Literature. Annual Review of Political Science, 7, 315-344.

Epstein, R. A. (2007). Defanging IRBs: Replacing Coercion with Information. Northwestern University Law Review, 101(2), 735-747.

Fitzgerald, M. H., Phillips, P. A., \& Yule, E. (2006). The Research Ethics Review Process and Ethics Review Narratives. Ethics \& Behavior, 16(4), 377-395.

Fraser, N. (1995). Toward a Discourse Ethic of Solidarity. Praxis International, 5(4), 425-429. 
Gastil, J., Black, L., \& Moscovitz, K. (2008). Ideology, Attitude Change, and Deliberation in Small Face-to-Face Groups. Political Communication, 25(1), 23-46.

Gunsalus, C. K., Bruner, E. M., Burbules, N. C., Dash, L., Finkin, M., Goldberg, J. P., et al. (2006). Mission Creep in the IRB World. Science, 312(5779), 1441.

Habermas, J. (1996). Between Facts and Norms (Studies in Contemporary German Social Thought). Cambridge: The MIT Press.

Hamburger, P. (2007). Getting Permission. Northwestern University Law Review, 101(2), 405492.

Judge, T. A., Bono, J. E., Ilies, R., \& Gerhardt, M. W. (2002). Personality and Leadership: A Qualitative and Quantitative Review. Journal of Applied Psychology, 87(4), 765-780.

Keith-Spiegel, P., \& Koocher, G. P. (2005). The IRB Paradox: Could the Protectors Also Encourage Deceit? Ethics \& Behavior, 15(4), 339-349.

Klitzman, R. L. (2011). The Myth of Community Differences as the Cause of Variation Among IRBs. AJOB Primary Research, 2(2), 24-33.

Klitzman, R. L. (2012a). Institutional Review Board Community Members: Who Are They, What Do They Do, and Whom Do They Represent? Academic Medicine, 87(7), 975-981.

Klitzman, R. L. (2012b). US IRBs Confronting Research in the Developing World. Developing World Bioethics, 12(2), 63-73.

Klitzman, R. L. (2013a). How IRB Leaders View and Approach Challenges Raised by IndustryFunded Research. IRB: Ethics and Human Research, 35(3), 9-16.

Klitzman, R. L. (2013b). How IRBs view and make decisions about coercion and undue influence. Journal of Medical Ethics, 39, 224-229.

Klitzman, R. L. (2013c). Views of IRBs Concerning Their Local Ecologies: Perceptions of Relationships, Systems, and Tensions Between IRBs and Their Institutions. AJOB Primary Research, 4(2), 31-43.

Kuyare, M. S., Marathe, P. A., Kuyare, S. S., \& Thatte, U. M. (2015). Perceptions and Experiences of Community Members Serving on Institutional Review Boards: A Questionnaire Based Study. HEC Forum, 27(1), 61-77.

Larson, E., Bratts, T., Zwanziger, J., \& Stone, P. (2006). A Survey of IRB Process in 68 U.S. Hospitals. Journal of Nursing Scholarship, 36(3), 260-264.

Latané, B., \& Wolf, S. (1981). The Social Impact of Majorities and Minorities. Psychological Review, 88(5), 438-453. 
Lidz, C. W., Appelbaum, P. S., Arnold, R. M., Candilis, P. J., Gardner, W., Myers, S., et al. (2012a). How Closely Do Institutional Review Boards Follow the Common Rule? Academic Medicine, 87(7), 969-974.

Lidz, C. W., Simon, L. J., Seligowski, A. V., Myers, S., Gardner, W., Candilis, P. J., et al. (2012b). The Participation of Community Members on Medical Institutional Review Boards. Journal of Empirical Research on Human Research Ethics, 7(1), 1-6.

London, A. J. (2012). A Non-Paternalistic Model of Research Ethics and Oversight: Assessing the Benefits of Prospective Review. Journal of Law, Medicine \& Ethics, 40(4), 930-944.

Maass, A., Ceccarelli, R., \& Rudin, S. (1996). Linguistic Intergroup Bias: Evidence for InGroup-Protective Motivation. Journal of Personality and Social Psychology, 71(3), 512526.

Mendelberg, T. (2002). The Deliberative Citizen: Theory and Evidence. In M. X. Delli Carpini, L. Huddy, \& R. Y. Shapiro (Eds.), Political Decision-Making, Deliberation and Participation (pp. 151-193). Greenwich, CT: Emerald Group Publishing.

National Health Service Health Research Authority (2012). Standard Operating Procedures for Research Ethics Committees. In National Research Ethics Service (Ed.), Version 5.1 (Vol. Version 5.1).

Porter, J. P. (1986). What Are the Ideal Characteristics of Unaffiliated/Nonscientist IRB Members? IRB: Ethics and Human Research, 8(3), 1-6.

Porter, J. P. (1987). How Unaffiliated/Nonscientist Members of Institutional Review Boards See Their Roles. IRB: Ethics and Human Research, 9(6), 1-6.

Rothstein, W. G., \& Phuong, L. H. (2007). Ethical Attitudes of Nurse, Physician, and Unaffiliated Members of Institutional Review Boards. Journal of Nursing Scholarship, 39(1), 75-81.

Ryfe, D. M. (2005). Does Deliberative Democracy Work? Annual Review of Political Science, $8(1), 49-71$.

Saver, R. S. (2005). What IRBs Could Learn from Corporate Boards. IRB: Ethics and Human Research, 27(5), 1-6.

Schuppli, C. A., \& Fraser, D. (2007). Factors Influencing the Effectiveness of Research Ethics Committees. Journal of Medical Ethics, 33(5), 294-301.

Sengupta, S., \& Lo, B. (2003). The Roles and Experiences of Nonaffiliated and Non-scientist Members of Institutional Review Boards. Academic Medicine, 78(2), 212-218.

Sunstein, C. (2003). The Law of Group Polarization. In J. Fishkin, \& P. Laslett (Eds.), Debating Deliberative Democracy (pp. 80-101). Malden, MA: Blackwell Publishing. 
Sunstein, C. (2006). Infotopia: How Many Minds Produce Knowledge. Oxford: Oxford University Press.

Sunstein, C. R. (2000). Deliberative Trouble? Why Groups Go to Extremes. Yale Law Journal, 110(1), 71-119.

U.S. Department of Health and Human Services (2009). 45 CFR 46 - Protection of Human Subjects. Accessed 2012-05-02.

Ulbert, C., \& Risse, T. (2005). Deliberately Changing the Discourse: What Does Make Arguing Effective? Acta Politica, 40(3), 351-367.

Whitney, S. N., Alcser, K., Schneider, C. E., McCullough, L. B., McGuire, A. L., \& Volk, R. J. (2008). Principal Investigator Views of the IRB System. International Journal of Medical Science, 5(2), 68-72. 